\title{
Enhanced Collection of Laboratory Data in HIV Surveillance Among 5 States with Confidential Name-based HIV Infection Reporting, 2005-2006
}

\author{
Kristen Mahle Gray ${ }^{*}, 1$, Tebitha Kajese ${ }^{2}$, Erin Crandell-Alden ${ }^{3}$, Bridget J. Anderson ${ }^{4}$, \\ Debbie Wendell ${ }^{5}$, Allison Crutchfield ${ }^{6}$, Terry Jackson ${ }^{7}$ and H. Irene Hall ${ }^{1}$, \\ for the Laboratory Reporting Workgroup ${ }^{\S}$
}

\author{
${ }^{I}$ Division of HIV/AIDS Prevention, National Center for HIV/AIDS, Viral Hepatitis, STD and TB Prevention, Centers for \\ Disease Control and Prevention, Atlanta, GA, USA \\ ${ }^{2}$ Business Computer Applications, 2002 Summit Blvd, Suite 880, Atlanta, GA, USA \\ ${ }^{3}$ HIV/STD/VH/TB Epidemiology Section, Division of Communicable Diseases, Bureau of Epidemiology, Michigan \\ Department of Community Health, Lansing, MI, USA \\ ${ }^{4}$ Bureau of HIV/AIDS Epidemiology, AIDS Institute, New York State Department of Health, Albany, NY, USA \\ ${ }^{5}$ HIV/AIDS Program, Louisiana Office of Public Health, New Orleans, LA, USA \\ ${ }^{6}$ Disease Control and Environmental Epidemiology Division, Colorado Department of Public Health and Environment, \\ Denver, CO, USA \\ ${ }^{7}$ Division of HIV/STD, Indiana State Department of Health, Indianapolis, IN, USA
}

\begin{abstract}
Laboratory data reported through HIV surveillance can provide information about disease severity and linkage to care; however these measures are only as accurate as the quality and completeness of data reported. Using data from five states that implemented enhanced collection of laboratory data in HIV surveillance from 2005-2006, we determined completeness of reporting, stage of disease at diagnosis, the most common opportunistic illnesses (OI) at diagnosis, and linkage to medical care. Methods to enhance laboratory reporting included increasing active surveillance efforts, identifying laboratories not reporting to HIV surveillance, increasing electronic reporting, and using laboratory results from auxiliary databases. Of 3,065 persons $\geq 13$ years of age diagnosed with HIV, 35.5\% were diagnosed with stage 3 (AIDS) and $37.7 \%$ progressed to stage 3 within 12-months after diagnosis. Overall, $78.5 \%$ were linked to care within 3 months; however, a higher proportion of persons with $\geq 1 \mathrm{CD} 4$ or viral load test was found among whites compared with blacks/African Americans $(82.1 \%$ vs $73.6 \%$, p $<0.001)$. Few $(12.3 \%)$ had an OI within 3 months of diagnosis. The completeness of laboratory data collected through surveillance was improved with enhanced reporting and provided a more accurate picture of stage of disease and gaps in linkage to care. Additional interventions are needed to meet the goals of the National HIV/AIDS Strategy on linkage to care and the reduction of HIV-related disparities.
\end{abstract}

Keywords: HIV diagnoses, HIV surveillance, CD4 and VL reporting, linkage to care.

\section{INTRODUCTION}

The clinical management of HIV disease relies on CD4+ T-lymphocyte (CD4) and plasma HIV-1 RNA (i.e., viral load) testing to guide the initiation of treatment and monitor care. The Department of Health and Human Services (DHHS) Panel on Antiretroviral Guidelines for Adults and Adolescents recommend CD4 count and viral load (VL) testing for a new patient during the initial visit and every 3 to 4 months after HIV diagnosis. Among patients who are clinically stable, CD4 may be monitored less frequently

*Address correspondence to this author at the HIV Incidence and Case Surveillance Branch, Division of HIV/AIDS Prevention, Centers for Disease Control and Prevention, Mailstop E-47, 1600 Clifton Road, NE, Atlanta, GA 30333, USA; Tel: 404-639-2050; Fax: 404-639-2980;

E-mail:blo9@cdc.gov

${ }^{\S}$ Laboratory Reporting Workgroup Participants: Elizabeth Hamilton ${ }^{3}$, Maria Kouznetsova $^{4}$ and Pamela Montoya ${ }^{6}$. (every 6-12 months) [1]. The reporting of CD4 and VL results to health departments enhances local and national HIV case surveillance data and is used to identify cases, stage disease at diagnosis, and monitor disease progression. CD4 and VL data can also be used to determine entry and retention in care, measure viral load suppression, and assess unmet healthcare needs; however these measures are only as accurate as the quality and completeness of data reported.

AIDS was a reportable condition by the mid-1980s in all 50 states and the District of Columbia; however AIDS surveillance was limited to the collection of clinical data. With the advent of antiretroviral medications, which have helped HIV-infected persons live longer, and the increased availability of HIV tests, the national focus has shifted to integrate AIDS surveillance and surveillance of HIV infection. In the early 1990s, surveillance programs began collecting CD4 test results as part of routine surveillance activities. This was in part a result of the expansion of the 
AIDS case definition in 1993 to include an immunologic definition of AIDS; CD4 counts of less than 200 cells $/ \mathrm{mm}^{3}$ or CD4 percentages less than $14 \%$ of total lymphocytes [2]. For the first time, the AIDS case definition could be met based exclusively on a positive HIV test and a low CD4 count or percentage. The most recent revision of the HIV surveillance case definition, in 2008, highlights the central role of CD4 results by using CD4 counts and percentages to define three stages of HIV infection, increasing in severity from stage 1 through stage 3 (AIDS) [3]. An unknown stage was built into the case definition to account for cases that do not have CD4 results or information on AIDS-defining conditions. The 2008 case definition also incorporated a new role for viral load test results in surveillance, as a detectable viral load became sufficient criteria for establishing a case for surveillance purposes [3].

The majority of U.S. states have policies or regulations that require laboratories to report $\mathrm{CD} 4$ and $\mathrm{VL}$ results to health departments. However, the level at which these laboratory results are reported varies within and across jurisdictions. In addition, barriers to maintaining complete and timely laboratory data in surveillance systems exist and may include the management of a large volume of reports and the receipt of paper $v s$ electronic reports.

We conducted enhanced data collection in five state HIV surveillance programs for CD4 and VL laboratory test results and OIs among newly diagnosed HIV-infected persons. Using these data, we evaluated the completeness of CD4 and VL laboratory reports collected through surveillance, determined stage of disease at diagnosis, the most common OIs reported at diagnosis, and linkage to medical care within 3 months of diagnosis using laboratory tests as a marker for receipt of care.

\section{MATERIALS AND METHODS}

Five surveillance jurisdictions (Colorado, Indiana, Louisiana, Michigan and New York [excluding New York City]) were selected to participate in the project as part of a competitive announcement. Analyses were based on data collected in the five states and reported to the Centers for Disease Control and Prevention (CDC) on HIV-infected persons aged 13 years or older diagnosed during April 2005 through March 2006, with the exception of New York, where persons were diagnosed from June 2005 through May 2006. Enhanced data collection of CD4 results, VL results, and OIs was conducted for all cases reported to the surveillance programs by 6 months after the end of the 12 month diagnosis period, except for Michigan where two out of three cases were sampled.

The five surveillance programs collected information according to routine surveillance procedures. Information was obtained on age, sex, race/ethnicity (white, black or African American, Hispanic or Latino, American Indian or Alaska Native, Asian, Native Hawaiian or Other Pacific Islander, and multiple races), transmission category (men who have sex with men [MSM], injection drug use [IDU], MSM and IDU [MSM/IDU], high-risk heterosexual contact, and other), CD4 result, VL result, and OI information. All analyses adjusted for reporting delays and missing risk factor information $[4,5]$.
Methods for the enhanced collection of data included: a) updating data in the surveillance software from existing auxiliary laboratory databases, b) strengthening active surveillance efforts through increased medical record abstraction of laboratory data, c) identifying and collecting data from laboratories not previously reporting to HIV surveillance, and d) increasing the number of laboratory reports being sent to the surveillance programs electronically. Enhanced medical record abstraction was conducted up to 6 months following the 12-month diagnosis period using the Adult HIV Case Report Form. Electronic and paper-based laboratory results received up to 6 months after the 12-month diagnosis period and all medical record data were entered into the surveillance software and transferred to $\mathrm{CDC}$ as part of routine reporting of national HIV surveillance data. Although there may have been differences in how reports were transmitted and collected across the states (e.g., passive transmission of paper or electronic reports from laboratories vs medical record abstraction), the goal was for each health department to receive all HIV-related laboratory data.

We determined the distribution of stage of disease at diagnosis based on CD4 results or OIs within 3 months of diagnosis and the number and percentage of persons diagnosed with HIV who were linked to care within 3,6 , and 12 months based on CD4 and VL tests within these time frames. We also determined the number and percentage of persons diagnosed with HIV who were linked to care within 3 months by demographic and transmission categories. Finally, we determined the OIs diagnosed within 3 months of HIV diagnosis overall and by level of immune-suppression (CD4 count $\geq 200$ cells $/ \mu \mathrm{L}$ or $\geq 14 \%$, and $<200$ cells $/ \mu \mathrm{L}$ or $<14 \%$ ).

To assess the impact of enhanced data collection, we compared the project data on two measures (stage of disease and linkage to care) with (1) data from the same five states on cases diagnosed within 12 months prior to the initiation of the project (population A), and (2) data from 25 states on cases diagnosed during April 2005 through March 2006 (population B). The 25 states were selected for the analysis because they met the criteria outlined in the Technical Guidance for HIV/AIDS Surveillance Programs of at least $50 \%$ of newly diagnosed persons having an initial CD4 or VL result within 3 months of diagnosis reported to the national HIV surveillance system. Population B did not include any of the states with enhanced data collection.

SAS software version 9.1 (SAS Institute., Cary, NC) was used to perform univariate and bivariate analyses with the $\mathrm{X}^{2}$ test. Chi-square p-values less than or equal to 0.05 were considered significant. To describe the completeness of data, we included all cases whether or not they were alive at the end of the observation period.

\section{RESULTS}

Of the 3,065 persons diagnosed during the 12-month diagnosis period in the five states, $9.6 \%$ were diagnosed with stage $1,29.1 \%$ with stage 2 , and $35.5 \%$ with stage 3 disease; $25.8 \%$ were stage unknown (Table 1A). The proportion of persons who had a result from at least one CD4 or VL increased as more time was allowed for tests to be performed 
Table 1A. Stage of HIV Infection Based on CD4 Test ${ }^{\text {a }}$ Performed within 3, 6, and 12 Months Following HIV Diagnosis Among Adults and Adolescents in 5 States $^{\mathrm{b}}$ with Enhanced Data Collection, 2005-2006

\begin{tabular}{|c|c|c|c|c|c|c|}
\hline & \multicolumn{2}{|c|}{$\leq 3 \mathrm{mo}$} & \multicolumn{2}{|c|}{$\leq 6 \mathrm{mo}$} & \multicolumn{2}{|c|}{$\leq 12 \mathrm{mo}$} \\
\hline Stage 2 & 891 & 29.1 & 968 & 31.6 & 1,031 & 33.6 \\
\hline Stage 3 (AIDS) & 1,087 & 35.5 & 1,120 & 36.5 & 1,155 & 37.7 \\
\hline Missing CD4 collection date & 1 & 0 & 0 & 0 & 0 & 0 \\
\hline Total & 3,065 & 100 & 3,065 & 100 & 3,065 & 100 \\
\hline
\end{tabular}

Table 1B. Stage of HIV Infection Based on CD4 Test ${ }^{a}$ Performed within 3, 6, and 12 Months Following HIV Diagnosis Among Adults and Adolescents in 5 States Before Enhanced Data Collection (Population $A^{c}$ ), 2004-2005

\begin{tabular}{|l|c|c|c|c|c|c|}
\hline & \multicolumn{2}{|c|}{$\leq \mathbf{3}$ mo } & \multicolumn{2}{|c|}{$\leq \mathbf{6}$ mo } & \multicolumn{2}{|c|}{ (\%) } \\
\cline { 2 - 7 } & No. & $\mathbf{( \% )}$ & No. & No. & 4.5 & 409 \\
\hline \hline Stage 1 & 331 & 7.6 & 374 & 26.2 & 1,271 & 29.0 \\
\hline Stage 2 & 1,027 & 23.4 & 1,149 & 35.0 & 1,586 & 36.2 \\
\hline Stage 3 (AIDS) & 1,465 & 33.4 & 1,531 & 30.3 & 1,113 & 25.4 \\
\hline Stage unknown & 1,556 & 35.5 & 1,326 & 0 & 0 & 0 \\
\hline Missing CD4 collection date & 0 & 0 & 0 & $\mathbf{1 0 0}$ & $\mathbf{4 , 3 7 9}$ & $\mathbf{1 0 0}$ \\
\hline Total & $\mathbf{4 , 3 7 9}$ & $\mathbf{1 0 0}$ & $\mathbf{4 , 3 7 9}$ & & 0 \\
\hline
\end{tabular}

Table 1C. Stage of HIV Infection Based on CD4 Test ${ }^{a}$ Performed within 3, 6, and 12 Months Following HIV Diagnosis Among Adults and Adolescents in 25 States without Enhanced Data Collection (Population $B^{d}$ ), 2005-2006

\begin{tabular}{|c|c|c|c|c|c|c|}
\hline & \multicolumn{2}{|c|}{$\leq 3 \mathrm{mo}$} & \multicolumn{2}{|c|}{$\leq 6 \mathrm{mo}$} & \multicolumn{2}{|c|}{$\leq 12 \mathrm{mo}$} \\
\hline Stage 2 & 2,104 & 19.8 & 2,305 & 21.7 & 2,518 & 23.7 \\
\hline Stage 3 (AIDS) & 3,412 & 32.1 & 3,575 & 33.6 & 3,743 & 35.2 \\
\hline Missing CD4 collection date & 2 & 0 & 1 & 0 & 1 & 0 \\
\hline Total & 10,631 & 100 & 10,631 & 100 & 10,631 & 100 \\
\hline
\end{tabular}

${ }^{\mathrm{a}}$ The lowest CD4 count or percentage taken from the time period of interest.

${ }^{b}$ Colorado, Indiana, Louisiana, Michigan (diagnosed between April 1, 2005 through March 31, 2006) and New York (diagnosed between June 1, 2005 and May 31, 2006).

${ }^{\mathrm{c}}$ Population A: Colorado, Indiana, Louisiana, Michigan (diagnosed between April 1, 2004 through March 31, 2005) and New York (diagnosed between June 1, 2004 and May 31, 2005).

${ }^{\mathrm{d}}$ Population B: Persons diagnosed with HIV in 25 states that had $\geq 50 \%$ of persons newly diagnosed between April 1, 2005 and March 31,2006 with an initial CD4 or VL result collected $\leq 3$ months of HIV diagnosis and reported to the national HIV surveillance system.

and reported (Table $\mathbf{2 A}$ ); however, the majority (78.5\%) had at least one CD4 or VL test within 3 months of diagnosis. Additionally, we assessed the impact of CD4 and VL tests initiated at the time of testing. When we removed persons who had the same diagnosis date and CD4 or VL collection date, the percentage of persons with a CD4 or VL test within 3 months dropped to $69.3 \%$, data not shown.
Blacks/African Americans were less likely to have a CD4 or VL test performed $(73.6 \%)$ within 3 months of HIV diagnosis than whites $(82.1 \%, \mathrm{p}<0.001)$ (Table 3). Persons aged 13-29 were also less likely to have a CD4 or VL test performed $(72.3 \%)$, as compared to persons aged 30 and above $(30-39$ years: $76.7 \%, 40-49$ years: $83.2 \%, \geq 50$ years: $86.6 \%$ ). Across the five states, there were significant differences in the percentage of persons with a result from at 
Table 2A. CD4 (Count or Percentage) and Viral Load Results Reported within 3, 6, and 12 Months Following HIV Diagnosis Among Adults and Adolescents in 5 States with Enhanced Data Collection, 2005-2006

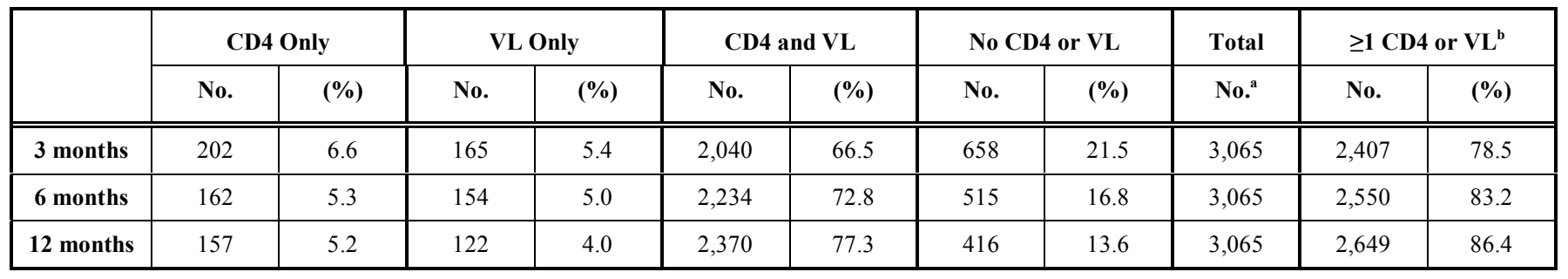

Table 2B. CD4 (Count or Percentage) and Viral Load Results Reported within 3, 6, and 12 Months Following HIV Diagnosis Among Adults and Adolescents in 5 States Before Enhanced Data Collection (Population A), 2004-2005

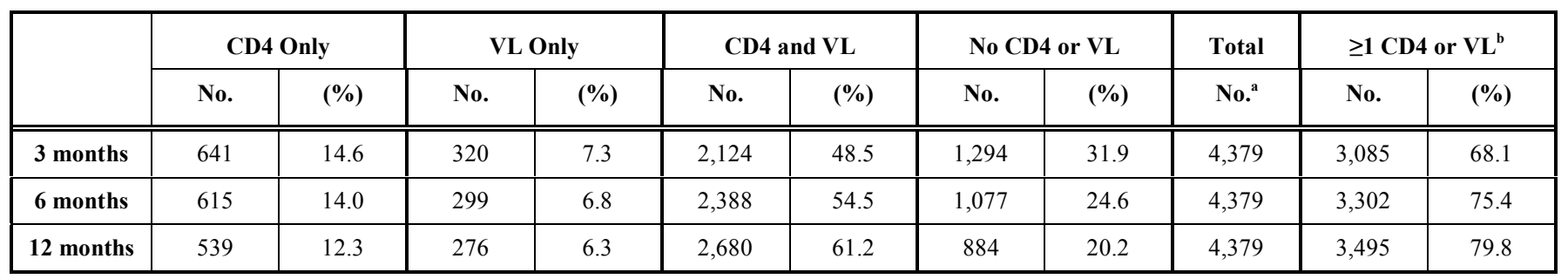

Table 2C. CD4 (Count or Percentage) and Viral Load Results Reported within 3, 6, and 12 Months Following HIV Diagnosis Among Adults And Adolescents in 25 States without Enhanced Data Collection (Population B), 2005-2006

\begin{tabular}{|c|c|c|c|c|c|c|c|c|c|c|c|}
\hline & \multicolumn{2}{|c|}{ CD4 Only } & \multicolumn{2}{|c|}{ VL Only } & \multicolumn{2}{|c|}{ CD4 and VL } & \multicolumn{2}{|c|}{ No CD4 or VL } & $\frac{\text { Total }}{\text { No. }^{\mathrm{a}}}$ & \multicolumn{2}{|c|}{$\geq 1 \mathrm{CD} 4$ or $\mathrm{VL}^{\mathrm{b}}$} \\
\hline 6 months & 2,321 & 21.8 & 822 & 7.7 & 5,094 & 47.9 & 2,390 & 22.5 & $10631^{\mathrm{c}}$ & 8,237 & 77.5 \\
\hline 12 months & 2,010 & 19.0 & 809 & 7.6 & 5,721 & 53.8 & 2,086 & 19.6 & $10631^{\mathrm{c}}$ & 8,540 & 80.4 \\
\hline
\end{tabular}

Note. CD4 = CD4+ T-lymphocyte count (cells/ $\mu \mathrm{L})$ or percentage; $\mathrm{VL}=$ viral load $(\operatorname{copies} / \mathrm{mL})$.

${ }^{a}$ Because column totals for estimated numbers were calculated independently of the values for the subpopulations, the values in each column may not sum to the column total.

${ }^{b}$ Data in this column $(\geq 1$ CD4 or VL) are not included in the column total.

${ }^{\mathrm{c}}$ Four cases did not have a CD4 or VL specimen collection date.

least one CD4 or VL test. The percentage of persons with a CD4 or VL test result also varied by the facility of diagnosis; $93.0 \%$ at adult HIV clinics to $52.9 \%$ at HIV counseling and testing sites. No significant differences were found between the percentage of persons with at least one CD4 or VL test result among males and females or across transmission categories.

The most frequently reported OI was Pneumocystis jiroveci pneumonia (PCP) $(38.6 \%, 197 / 511)$, followed by Esophageal Candidiasis $(16.4 \%, 84 / 511)$, and Wasting Syndrome $(7.2 \%, 37 / 511)$ (Table 4). Of the 378 persons with an OI diagnosed within 3 months of HIV diagnosis, a larger percentage of OIs was found among persons with a CD4 result of $<200$ cells $/ \mu \mathrm{L}$ or $<14 \%(98.3 \%)$ as compared to persons with a CD4 result of $\geq 200$ cells $/ \mu \mathrm{L}$ or $\geq 14 \%$ (1.7\%).

Enhanced data collection appears to have resulted in more complete data and therefore a more accurate measurement of stage of disease and linkage to care. The proportion of persons classified as stage unknown within 12 months of diagnosis was larger in the comparison populations, with $25.4 \%$ and $30.3 \%$ in Population A and B, respectively, compared to $17.0 \%$ among cases with enhanced data collection (Table 1). As the time between HIV diagnosis and the first CD4 test performed increased from 3 months to 6 and 12 months, the percentage of persons classified as stage unknown decreased in all populations.

Persons in the five states with enhanced data collection (Table 2A) were more likely to have at least one CD4 or VL test result at 12 months after diagnosis $(86.4 \%)$ compared with persons in Population A $(79.8 \%, \mathrm{p}<0.001$, Table 2B) or Population B (80.4\%, $\mathrm{p}<0.001$, Table 2C). Within 3 months of HIV diagnosis, $78.5 \%$ of persons with enhanced data collection had a result from at least one CD4 or VL test, as compared to $68.1 \%$ and $74.7 \%$ in Population A and B respectively. Persons in the five states were also more likely to have an OI reported to surveillance within 3 months of diagnosis during the time of enhanced data collection than during the previous year (Population A) $(12.3 \%$ vs $10.6 \%$, $\mathrm{p}=0.02$ ), data not shown.

\section{DISCUSSION}

Enhanced collection of laboratory data through the utilization of data in existing databases, strengthened active surveillance efforts, the identification of labs not reporting to surveillance, and an increased number of electronic reports, improved the completeness of $\mathrm{CD} 4, \mathrm{VL}$, and $\mathrm{OI}$ data in national HIV surveillance. Although no true standard was 
Table 3. CD4 (Count or Percentage) and Viral Load Results Reported within 3 Months Following HIV Diagnosis Among Adults and Adolescents in 5 States with Enhanced Data Collection, by Selected Characteristics, 2005-2006

\begin{tabular}{|c|c|c|c|c|c|c|c|}
\hline & CD4 Only & VL Only & CD4 and VL & $\begin{array}{c}\text { No CD4 or } \\
\text { VL }\end{array}$ & Total $^{\mathrm{a}}$ & $\geq 1 \mathrm{CD} 4$ or $\mathrm{VL}^{\mathrm{c}}$ & \multirow[t]{2}{*}{$\begin{array}{c}\geq 1 \text { CD4 or VL } \\
\text { Chi-Square } \\
\text { p-Value }\end{array}$} \\
\hline & No. (\%) & No. $(\%)$ & No. $(\%)$ & No. $(\%)$ & No. $(\%)^{\text {b }}$ & No. $(\%)$ & \\
\hline \multicolumn{8}{|l|}{ Sex } \\
\hline Male & $149(6.8)$ & $112(5.1)$ & $1,454(66.1)$ & $484(22.0)$ & $2,199(71.7)$ & $1,715(78.0)$ & Reference \\
\hline Female & $53(6.1)$ & $53(6.2)$ & $586(67.6)$ & $174(20.1)$ & $866(28.3)$ & $692(79.9)$ & 0.25 \\
\hline \multicolumn{8}{|l|}{ Race/Ethnicity } \\
\hline Black/African American & $117(8.0)$ & $60(4.1)$ & $900(61.5)$ & $386(26.4)$ & $1,463(47.7)$ & $1,077(73.6)$ & $<0.001$ \\
\hline Hispanic/Latino & $18(5.1)$ & $16(4.5)$ & $267(73.6)$ & $61(16.9)$ & $362(11.8)$ & $301(83.1)$ & 0.99 \\
\hline White & $51(4.8)$ & $75(7.1)$ & $755(71.3)$ & $179(16.9)$ & $1,059(34.6)$ & $880(82.1)$ & Reference \\
\hline Multiple races/Other race $^{d}$ & $16(8.8)$ & $14(7.7)$ & $118(65.4)$ & $33(18.1)$ & $181(5.9)$ & $148(81.9)$ & 0.69 \\
\hline \multicolumn{8}{|l|}{ Age } \\
\hline $13-29$ & $49(5.3)$ & $45(4.8)$ & $575(62.1)$ & $257(27.7)$ & $926(30.2)$ & $669(72.3)$ & 0.03 \\
\hline $30-39$ & $56(6.3)$ & $50(5.6)$ & $579(64.9)$ & $208(23.3)$ & $893(29.1)$ & $685(76.7)$ & Reference \\
\hline $40-49$ & $62(7.6)$ & $44(5.4)$ & $571(70.3)$ & $136(16.8)$ & $813(26.5)$ & $677(83.2)$ & $<0.001$ \\
\hline$\geq 50$ & $34(7.9)$ & $27(6.2)$ & $315(72.5)$ & $58(13.4)$ & $434(14.2)$ & $376(86.6)$ & $<0.001$ \\
\hline \multicolumn{8}{|l|}{ Transmission Category } \\
\hline \multicolumn{8}{|l|}{ Male Adult or Adolescent } \\
\hline Male-to-male sexual contact & $86(5.5)$ & $79(5.0)$ & $1058(67.5)$ & $344(21.9)$ & $1,567(71.3)$ & $1,223(78.1)$ & Reference \\
\hline Injection drug use & $23(11.8)$ & $8(4.4)$ & $116(60.7)$ & $44(23.1)$ & $191(8.7)$ & $147(76.9)$ & 0.71 \\
\hline Male-to-male sexual contact and injection drug use & $17(10.8)$ & $5(3.4)$ & $103(66.3)$ & $30(19.5)$ & $156(7.1)$ & $126(80.5)$ & 0.49 \\
\hline Heterosexual contact $^{\mathrm{e}}$ & $21(7.6)$ & $18(6.4)$ & $171(62.1)$ & $66(24.0)$ & $275(12.5)$ & $209(76.0)$ & 0.45 \\
\hline Other & $2(21.6)$ & $1(14.7)$ & $6(62.6)$ & 0 & $10(0.4)$ & $10(100.0)$ & f \\
\hline \multicolumn{8}{|l|}{ Female Adult or Adolescent } \\
\hline Injection drug use & $12(7.2)$ & $14(8.3)$ & $118(69.1)$ & $26(15.4)$ & $171(19.8)$ & $145(84.6)$ & 0.07 \\
\hline Heterosexual contact & $39(5.8)$ & $39(5.7)$ & $458(67.0)$ & $148(22.4)$ & $684(79.0)$ & $536(77.6)$ & Reference \\
\hline Other & $1(13.5)$ & 0 & $9(82.6)$ & 0 & $11(1.2)$ & $11(100.0)$ & $\mathrm{f}$ \\
\hline \multicolumn{8}{|l|}{ Project Area } \\
\hline Colorado & $24(5.3)$ & $16(3.6)$ & $338(76.7)$ & $63(14.3)$ & $440(14.4)$ & $377(85.7)$ & $<0.001$ \\
\hline Indiana & $28(5.9)$ & $19(4.1)$ & $307(65.5)$ & $114(24.4)$ & $468(15.3)$ & $354(75.6)$ & 0.61 \\
\hline Louisiana & $89(10.0)$ & $30(3.3)$ & $568(63.5)$ & $208(23.2)$ & $895(29.2)$ & $687(76.8)$ & Reference \\
\hline Michigan & $41(7.6)$ & $17(3.2)$ & $327(60.6)$ & $154(28.6)$ & $540(17.6)$ & $386(71.4)$ & 0.02 \\
\hline New York & $20(2.7)$ & $83(11.5)$ & $500(69.3)$ & $119(16.5)$ & $722(23.5)$ & $603(83.5)$ & $<0.001$ \\
\hline \multicolumn{8}{|l|}{ Facility of Diagnosis } \\
\hline Private physician/HMO & $20(4.2)$ & $30(6.3)$ & $346(73.1)$ & $78(16.5)$ & $474(15.5)$ & $396(83.5)$ & 0.08 \\
\hline Emergency room/Inpatient clinic & $87(13.3)$ & $27(4.2)$ & $457(69.7)$ & $84(12.9)$ & $656(21.4)$ & $571(87.1)$ & Reference \\
\hline Adult HIV clinic & $10(8.0)$ & $6(5.0)$ & $104(79.8)$ & $9(7.2)$ & $130(4.2)$ & $120(93.0)$ & 0.008 \\
\hline HIV counseling and testing site & $18(5.2)$ & $13(3.7)$ & $151(44.0)$ & $161(47.1)$ & $342(11.2)$ & $181(52.9)$ & $<0.001$ \\
\hline STD clinic & $14(11.4)$ & $2(1.8)$ & $54(45.1)$ & $50(41.7)$ & $119(3.9)$ & $69(58.3)$ & $<0.001$ \\
\hline Correctional facility & $4(4.1)$ & $4(4.2)$ & $66(65.0)$ & $27(26.6)$ & $102(3.3)$ & $75(73.4)$ & 0.01 \\
\hline Other clinic & $17(4.0)$ & $20(4.9)$ & $230(55.1)$ & $150(36.0)$ & $417(13.6)$ & $267(63.9)$ & $<0.001$ \\
\hline Unknown & $32(3.9)$ & $62(7.6)$ & $633(76.7)$ & $98(11.9)$ & $825(26.9)$ & 727 (88.1) & 0.02 \\
\hline Total & $202(6.6)$ & $165(5.4)$ & $2,040(66.6)$ & $659(21.5)$ & 3,065 & $2,406(78.5)$ & \\
\hline
\end{tabular}

Note. CD4 = CD4+ T-lymphocyte count (cells $/ \mu \mathrm{L})$ or percentage; $\mathrm{VL}=$ viral load (copies $/ \mathrm{mL})$.

${ }^{\mathrm{a} B}$ Because column totals for estimated numbers were calculated independently of the values for the subpopulations, the values in each column may not sum to the column total.

${ }^{\mathrm{b}}$ The total percent represents the column percent.

${ }^{\mathrm{c}}$ Data in this column $(\geq 1 \mathrm{CD} 4$ or $\mathrm{VL})$ are not included in the column total.

dOther race: American Indian/Alaska Native, Asian, or Native Hawaiian/Other Pacific Islander

${ }^{\mathrm{e}}$ Heterosexual contact with a person known to have, or to be at high risk for, HIV infection.

${ }^{\mathrm{f}}$ The Chi-square test is invalid due to small cell size.

available for comparison, the percentages of persons diagnosed with HIV who had stage of disease assigned or who were linked to care were up to $10 \%$ higher with the enhanced data collection. 
Table 4. Opportunistic Illnesses Reported within 3 Months Following HIV Diagnosis Among Adults and Adolescents in 5 States with Enhanced Data Collection, by CD4 Result, 2005-2006

\begin{tabular}{|c|c|c|c|c|c|c|c|}
\hline \multirow[b]{2}{*}{ Overall OIs } & \multicolumn{2}{|c|}{ CD4 Count $\geq 200$ Cells $/ \mu \mathrm{L}$ or $\geq 14 \%$} & \multicolumn{2}{|c|}{ CD4 Count $<200$ Cells $/ \mu \mathrm{L}$ or $<14 \%$} & \multicolumn{2}{|c|}{ No CD4 Result } & \multirow{2}{*}{\begin{tabular}{|c|} 
Total \\
No.
\end{tabular}} \\
\hline & No. & $\%$ & No. & $\%$ & No. & $\%$ & \\
\hline OI within 3 months & 6 & 1.7 & 372 & 98.3 & 0 & 0 & 378 \\
\hline OI with incomplete diagnosis date (missing mo.) & 0 & 0 & 1 & 100 & 0 & 0 & $1^{\mathrm{c}}$ \\
\hline No OI within 3 months & 1,179 & 43.9 & 714 & 26.5 & 792 & 29.5 & 2,686 \\
\hline Total & 1,185 & 1,087 & 792 & 3,065 & & & \\
\hline Pneumocystis jiroveci pneumonia & 1 & 0.5 & 196 & 99.5 & 0 & 0 & 197 \\
\hline Candidiasis, Esophageal & 1 & 1.3 & 83 & 98.7 & 0 & 0 & 84 \\
\hline Wasting Syndrome & 0 & 0 & 37 & 100 & 0 & 0 & 37 \\
\hline Candidiasis of Bronchi, Trachea, or Lungs & 0 & 0 & 12 & 100 & 0 & 0 & 12 \\
\hline Cryptococcosis & 0 & 0 & 16 & 100 & 0 & 0 & 16 \\
\hline Histoplasmosis & 0 & 0 & 14 & 100 & 0 & 0 & 14 \\
\hline M. tuberculosis, Disseminated or Extrapulmonary & 0 & 0 & 12 & 100 & 0 & 0 & 12 \\
\hline Encephalopathy, HIV-related & 0 & 0 & 11 & 100 & 0 & 0 & 11 \\
\hline Herpes simplex & 0 & 0 & 11 & 100 & 0 & 0 & 11 \\
\hline Mycobacterium avium complex or M. kansasii & 0 & 0 & 11 & 100 & 0 & 0 & 11 \\
\hline Kaposi's Sarcoma & 0 & 0 & 16 & 100 & 0 & 0 & 16 \\
\hline Pneumonia, Recurrent & 1 & 10.1 & 10 & 89.9 & 0 & 0 & 11 \\
\hline Lymphoma, Primary, of Brain & 0 & 0 & 2 & 100 & 0 & 0 & 2 \\
\hline Mycobacterium, Other Species & 0 & 0 & 2 & 100 & 0 & 0 & 2 \\
\hline Progressive Multifocal Leukoencephalopathy & 0 & 0 & 1 & 100 & 0 & 0 & 1 \\
\hline Total $^{\mathrm{b}}$ & 8 & 1.6 & 503 & 98.4 & 0 & 0 & 511 \\
\hline
\end{tabular}

Note. $\mathrm{CD} 4=\mathrm{CD} 4+\mathrm{T}$-lymphocyte count $($ cells $/ \mu \mathrm{L})$ or percentage.

${ }^{a}$ The following OIs were not represented in this population: Cervical Cancer (Invasive), Isosporiasis, Salmonella Septicemia (adult only).

${ }^{b}$ The total individual OIs do not add up to the total OIs $(n=378)$, as some cases were diagnosed with more than one OI.

${ }^{\mathrm{c}}$ One case had a missing CD4 specimen collection date and could not be categorized by CD4 result.

The collection of more complete data provides a more accurate picture of disease severity and linkage to care. Based on CD4 and VL laboratory tests performed within 3 months following diagnosis, we estimated that $78.5 \%$ of persons diagnosed in the five states were linked to care. This finding is somewhat higher than what was observed through an analysis of persons newly diagnosed with HIV infection in New York City, where the time between the first positive Western blot test and the first CD4 and/or VL result reported to surveillance was used to indicate the time period from the initial HIV diagnosis (non-AIDS) to the first HIV-related medical care visit [6]. Of 1,928 newly diagnosed persons in
New York City in 2003, an estimated $63.7 \%$ initiated care within 3 months of diagnosis.

Overall, linkage to care needs strengthening to reach the goal outlined in the National HIV/AIDS Strategy of $85 \%$ of newly diagnosed patients linked to clinical care within 3 months of diagnosis [7]. We found a significantly higher proportion of persons with no CD4 or VL test results among blacks/African Americans as compared to whites which suggests that a racial disparity may exist among newly diagnosed persons who receive care. This finding is consistent with other studies that have documented an 
association between racial factors and disparities in HIVrelated healthcare [8-11] and may be related to a complex interaction between health care, public health, and social factors [12]. These data also show that younger persons aged 15-29 were less likely to have a CD4 or VL test, as compared to persons aged 30 and above. The disparity seen in age may be a function of the large proportion of young adults that are uninsured [13], and serves to highlight the potential gap in accessing care between age groups. Additional interventions are needed to meet the goals of the National HIV/AIDS Strategy on linkage to care and the reduction of HIV-related disparities.

The initiation and frequency of laboratory testing and interpretation of laboratory results can be used to make inferences about the quality of health care that HIV-infected persons receive. Laboratory testing that occurs shortly after HIV diagnosis implies the successful linkage to health care. Serial CD4 and VL testing suggests utilization of ongoing care compared with a one-time visit. If the frequency of serial laboratory tests meet clinical management testing guidelines (e.g., every 3-4 months after initial diagnosis baseline measurement), it suggests receipt of higher quality care than less frequent laboratory testing would.

Persons without CD4 or VL testing following HIV diagnosis may represent persons with unmet healthcare needs. To address unmet healthcare needs and develop effective interventions, it is important to understand the barriers to accessing care such as lack of health insurance coverage $[14,15]$, unsuccessful patient notification of positive HIV test results [16-18], denial, poverty, mental illness, lack of transportation, and homelessness [19-21]. States have a responsibility to provide unmet health care need estimates to the Health Resources and Services Administration (HRSA), which oversees the Ryan White CARE Act. Surveillance data could be used to provide estimates of persons not in care and, therefore, assist states in meeting this federal reporting obligation.

OI data can be a useful indicator of clinical outcomes for $\mathrm{HIV}$-infected persons in care. Based on OI data reported within 3 months following diagnosis, we estimated that $12.3 \%$ of persons diagnosed in the five states had at least one OI; PCP was the most frequently reported. A similar finding was observed through an analysis of a population-based survey of persons in HIV-related medical care in King County Washington, selected health districts in Louisiana, and the state of Michigan [22]. Of all HIV-infected persons in care in these areas in 1998, 11.3\% (CI, 8.8-13.9) had at least one OI diagnosis and PCP was the most commonly diagnosed.

There are some limitations to consider when interpreting our findings. First, if laboratory data reported to surveillance are incomplete, the methods outlined may underestimate the prevalence of persons with access to medical care. In addition, the contribution of individuals who may be diagnosed in a jurisdiction reported to a state HIV program but then leave the state is unknown. From the local surveillance perspective, these individuals may not have begun care (i.e., evidence of lab testing) but received care after they moved out of jurisdiction. In Louisiana, the number of cases reported to surveillance and the completeness of laboratory data collected after August 2005 was impacted by Hurricane Katrina, as several clinical sites were closed and complete medical record abstraction was not possible.

In jurisdictions that have laws or regulations for laboratory reporting of all HIV-related tests, private and public laboratories must report all test results to the respective health departments. During the data collection period, changes in CD4 and VL reporting laws may have contributed to improvements seen in the completeness of laboratory reporting. For example, the laboratory reporting requirements were significantly broadened in New York State in June 2005, the start of the 12-month diagnosis period, to require the reporting of any VL result and all CD4 counts and percentages. Prior to this change, only detectable VLs and CD4 counts less than 500 cells $/ \mu \mathrm{L}$ or percentages less than $29 \%$ were reportable. In July 2005, Michigan also revised their regulations to require the reporting of all CD4 and VL results.

In general, surveillance programs believe they are receiving the vast majority of HIV test results; but, unless a state is actively monitoring the number of laboratories reporting to them and the volume of reports, they cannot know for certain. Instances where laboratory testing may change (e.g., the provider decides to use another laboratory or the primary laboratory contracts with a new laboratory for specialized testing) may not necessarily be identified by a surveillance program. Active surveillance and medical record abstraction can help to obtain more complete laboratory data and identify laboratories that may not be reporting to HIV surveillance. The electronic transmission of HIV-related laboratory test results enhances the completeness, timeliness, and accuracy of reporting to surveillance programs [23]. Although many surveillance programs have received data electronically for years, many still need improvements or enhancements to implement and maintain the system. HIV surveillance programs are currently using software called eHARS that has the capacity for storage of all laboratory results and the ability for electronic laboratory data to be imported. To ensure that all laboratory results are reported to surveillance at the national level, state and local surveillance programs must ensure that all laboratory results, including those stored in auxiliary databases, are entered into eHARS software and transmitted to $\mathrm{CDC}$.

The National HIV/AIDS Strategy proposes to reduce new HIV infections, increase access to care and improve health outcomes for people living with HIV, and reduce HIVrelated disparities [8]. Data collected through the national HIV surveillance system can be used to monitor the outcomes of the national strategy; however the validity of these measures is dependent upon the completeness and quality of surveillance data. Monitoring outcomes such as linkage to care and racial/ethnic disparities among persons who are virally suppressed is particularly dependent upon complete reporting of all HIV-related laboratory results to surveillance.

\section{ACKNOWLEDGEMENTS}

The authors would like to acknowledge Elizabeth Hamilton $^{\mathrm{a}}$, Maria Kouznetsova ${ }^{\mathrm{b}}$, and Pamela Montoya ${ }^{\mathrm{c}}$ of 
the Laboratory Reporting Workgroup for their contribution to this project.

${ }^{\mathrm{a}} \mathrm{HIV} / \mathrm{STD} / \mathrm{VH} / \mathrm{TB}$ Epidemiology Section, Division of Communicable Diseases, Bureau of Epidemiology, Michigan Department of Community Health, Lansing, MI, USA.

${ }^{b}$ Bureau of HIV/AIDS Epidemiology, AIDS Institute, New York State Department of Health, Albany, NY, USA.

${ }^{c}$ Disease Control and Environmental Epidemiology Division, Colorado Department of Public Health and Environment, Denver, CO, USA.

\section{CONFLICT OF INTEREST}

None of the authors have a reported financial, consultant, institutional or other conflict of interest in the publication of this manuscript.

\section{DISCLAIMER}

The findings and conclusions in this report are those of the authors and do not necessarily represent the official view of the Centers for Disease Control and Prevention

\section{REFERENCES}

[1] Panel on Antiretroviral Guidelines for Adults and Adolescents. Guidelines for the Use of Antiretroviral Agents in HIV-1-Infected Adults and Adolescents. January 10, 2011. Rockville, MD: Department of Health and Human Services; 2009. Available at: http://aidsinfo.nih.gov/contentfiles/AdultandAdolescentGL.pdf [Accessed: December 7, 2011]

[2] Centers for Disease Control and Prevention. Revised classification system for HIV infection and expanded surveillance case definition for AIDS among adolescents and adults, 1993. MMWR Morb Mortal Wkly Rep 1992; 41(RR-17): 1-19.

[3] Centers for Disease Control and Prevention. Revised surveillance case definitions for HIV infection among adults, adolescents, and children aged $<18$ months and for HIV infection and AIDS among children aged 18 months to $<13$ years--United States, 2008. MMWR Morb Mortal Wkly Rep 2008; 57(RR-10): 1-12.

[4] Song R, Hall HI, Frey R. Uncertainties associated with incidence estimates of HIV/AIDS diagnoses adjusted for reporting delay and risk redistribution. Stat Med 2005; 24(3): 453-64.

[5] McDavid HK, Kajese T, Hall HI, Song R. Risk factor redistribution of the national HIV/AIDS surveillance data: an alternative approach. Public Health Rep 2008; 123(5): 618-27.

[6] Torian LV, Wiewel EW, Liu KL, Sackoff JE, Frieden TR. Risk factors for delayed initiation of medical care after diagnosis of human immunodeficiency virus. Arch Intern Med 2008; 168(11): 1181-7.
[7] Office of National AIDS Policy. National HIV/AIDS Strategy. Washington, DC: Office of National AIDS Policy; 2010. Available at: http://www.whitehouse.gov/administration/eop/onap/nhas [Accessed: February 25, 2011]

[8] Gebo KA, Fleishman JA, Conviser R, et al. Racial and gender disparities in receipt of highly active antiretroviral therapy persist in a multistate sample of HIV patients in 2001. J Acquir Immune Defic Syndr 2005; 38(1): 96-103.

[9] Keruly JC, RD Moore. Immune status at presentation to care did not improve among antiretroviral-naive persons from 1990 to 2006. Clin Infect Dis 2007; 45(10): 1369-74.

[10] Mugavero MJ, Lin HY, Allison JJ, et al. Racial disparities in HIV virologic failure: do missed visits matter? J Acquir Immune Defic Syndr 2009; 50(1): 100-8.

[11] Ulett KB, Willig JH, Lin HY. The therapeutic implications of timely linkage and early retention in HIV care. AIDS Patient Care STDS 2009; 23(1): 41-9.

[12] Lurie N, Dubowitz T. Health disparities and access to health JAMA 2007; 297(10): 1118-21.

[13] Voelker R. Health reforms called a "graduation gift" for young adults who lack insurance. JAMA 2010; 304(2): 147.

[14] Turner BJ, Cunningham WE, Duan N, et al. Delayed medical care after diagnosis in a US national probability sample of persons infected with human immunodeficiency virus. Arch Intern Med 2000; 160(17): 2614-22.

[15] Stein MD, Crystal S, Cunningham WE, et al. Delays in seeking HIV care due to competing caregiver responsibilities. Am J Public Health 2000; 90(7): 1138-40.

[16] Eichler MR, Ray SM, del Rio C. The effectiveness of HIV post-test counseling in determining healthcare-seeking behavior. AIDS 2002; 16(6): 943-5.

[17] Sullivan PS, Lansky A, Drake A. Failure to return for HIV test results among persons at high risk for HIV infection. J Acquir Immune Defic Syndr 2004; 35(5): 511-8.

[18] Hightow LB, Miller WC, Leone PA, Wohl D, Smurzynski M, Kaplan AH. Failure to return for HIV posttest counseling in an STD clinic population. AIDS Educ Prev 2003; 15(3): 282-90.

[19] Raveis VH, Siegel K, Gorey E. Factors associated with HIVinfected women's delay in seeking medical care. AIDS Care 1998; 10(5): 549-62.

[20] Konkle-Parker DJ, Amico KR, Henderson HM. Barriers and facilitators to engagement in HIV clinical care in the Deep South: results from semi-structured patient interviews. J Assoc Nurses AIDS Care 2011; 22(2): 90-9.

[21] Horstman E, Brown J, Islam F, et al. Retaining HIV-Infected Patients in Care: Where Are We? Where Do We Go from Here? Clin Infec Dis 2010; 50: 752-61.

[22] Sullivan PS, Denniston M, McNaghten A, Buskin SE, Broyles ST, Mokotoff ED. Use of a population-based survey to determine incidence of AIDS-defining opportunistic illnesses among HIVpositive persons receiving medical care in the United States. AIDS Res Ther 2007; 12; 4: 17.

[23] Effler P, Ching-Lee M, Bogard A, et al. Statewide system of electronic notifiable disease reporting from clinical laboratories. JAMA 1999; 282: 1845-50. 